\title{
Editorial: Plasticity of Endogenous Pain Modulatory Circuits in Neuropathy
}

\author{
Ryan Patel ${ }^{1 *}$, Bridget M. Lumb ${ }^{2}$ and Kirsty Bannister ${ }^{1}$ \\ ${ }^{1}$ Wolfson Centre for Age Related Diseases, King's College London, London, United Kingdom, ${ }^{2}$ School of Physiology, \\ Pharmacology and Neuroscience, University of Bristol, Bristol, United Kingdom
}

Keywords: neuropathic pain, endogenous pain modulation, descending pain control, animal models, fMRI, chronic pain, monoamines, endocannabinoids

\section{Editorial on the Research Topic}

Plasticity of Endogenous Pain Modulatory Circuits in Neuropathy

\section{BACKGROUND}

Acute pain has a physiological role to protect against tissue damage, however chronic pain can persist as a sequela of a multitude of diseases due to maladaptive plasticity in the peripheral and central nervous system. New treatment algorithms are desperately needed as it is clear that when treated as a homogenous patient population many fail to manage their pain effectively $(1,2)$.

Early seminal studies demonstrated that micro-stimulation of the mid-brain produced a profound analgesia (3). It was later shown that this modulation is bi-directional and serves

\section{OPEN ACCESS}

Edited and reviewed by: Albert Dahan

Leiden University Medical Center, Netherlands

*Correspondence:

Ryan Patel

ryan.pate/@kcl.ac.uk

Specialty section: This article was submitted to Neuropathic Pain, a section of the journal

Frontiers in Pain Research

Received: 14 September 2021 Accepted: 24 September 2021

Published: 21 October 2021

Citation:

Patel R, Lumb BM and Bannister K (2021) Editorial: Plasticity of Endogenous Pain Modulatory Circuits in Neuropathy.

Front. Pain Res. 2:776948. doi: 10.3389/fpain.2021.776948 to amplify or suppress spinal sensory transmission. At rest these descending controls are balanced to fine-tune sensory gain but can rapidly adapt depending on context, expectation, and emotional state. Key examples include placebo analgesia, offset analgesia/onset hyperalgesia, attentional analgesia, stress-induced analgesia/hyperalgesia, and conditioned pain modulation. Each of these co-exist within the descending pain modulatory system (DPMS) and depend on partially overlapping signalling systems and circuits. For example, placebo analgesia in part requires descending opioidergic pathways (4), conditioned pain modulation is mediated by mainly noradrenergic and partly opioidergic signalling $(5,6)$, whereas attentional analgesia, stress-induced analgesia/hyperalgesia and offset analgesia are associated with the periaqueductal grey-dorsal raphe axis (7-9).

There is an extensive body of pre-clinical evidence that links altered descending control to the development and maintenance of neuropathic pain states (10-13), and these findings are supported by clinical studies based on imaging or psychophysical tests. The most widely studied in patients is conditioned pain modulation (CPM), also referred to as diffuse noxious inhibitory controls, which may represent a translatable endpoint linking pre-clinical and clinical investigations (14). This form of dynamic quantitative sensory testing is often simplified as "pain inhibits pain" where two distant noxious stimuli produce analgesia, and likely represents a surrogate measure of the net balance of descending facilitatory and inhibitory signalling. Inefficient CPM provides an insight into pathophysiological mechanisms and has been observed in neuropathic and chronic pain conditions $(6,15-17)$. Inefficient CPM has also been linked to "pain vulnerability" for post-operative pain $(18,19)$, and could be useful as a sensory biomarker for mechanism-led treatment selection as tapentadol/duloxetine efficacy are inversely correlated with CPM efficiency in neuropathic patients $(6,20)$. 
Clearly there is still much to be learned about the mechanisms and circuits that subserve different aspects of the DPMS. This may ultimately help define individual differences in pain mechanisms, pain vulnerability, and guide treatment selection. Within this Research Topic we collated original research and review articles providing novel insight into the function of the DPMS in neuropathic pain.

\section{OVERVIEW OF THE ARTICLES INCLUDED IN THIS RESEARCH TOPIC}

In their original research article Osborne et al. perform a longitudinal study to examine differences in subgenual anterior cingulate cortex (sgACC) functional connectivity in patients with carpel tunnel syndrome and those that recovered after surgery. The sgACC is thought to engage structures within the DPMS such as the periaqueductal grey, dorsal raphe, and prefrontal cortex implicating a role in endogenous pain modulation. In healthy individuals, sex differences have been observed in resting functional connectivity; women had greater connectivity with the DPMS pathways (periaqueductal grey and raphe nuclei) whereas men showed greater engagement of salience networks. In carpel tunnel syndrome, sgACC functional connectivity with the prefrontal cortex and temporal lobes was found to be reduced in men but not women when compared to healthy controls. In addition to sex differences in the dynamic connectome, plasticity in sgACC functional connectivity was observed following surgical treatment. Prior to surgery, patients had stronger sgACC functional connectivity with the orbitofrontal cortex, dorsal striatum, premotor cortex, and middle frontal gyrus compared with after surgery. After surgery patients had greater sgACC connectivity with the posterior insula and central/parietal operculum compared with their pre-operative scans.

In their original research article Boullon et al., studied sex differences in the development of neuropathic pain in a rat model of traumatic nerve injury and associated levels of endocannabinoids. Female rats exhibited earlier onset of hypersensitivity and increased sensitivity to cold and mechanical stimulation. Co-morbidities were also examined

\section{REFERENCES}

1. Breivik H, Collett B, Ventafridda V, Cohen R, Gallacher D. Survey of chronic pain in Europe: prevalence, impact on daily life, and treatment. Eur J Pain. (2006) 10:287-333. doi: 10.1016/j.ejpain.2005.0 6.009

2. Finnerup NB, Attal $\mathrm{N}$, Haroutounian $\mathrm{S}$, McNicol E, Baron $\mathrm{R}$, Dworkin RH, et al. Pharmacotherapy for neuropathic pain in adults: a systematic review and meta-analysis. Lancet Neurol. (2015) 14:162-73. doi: 10.1016/S1474-4422(14)70251-0

3. Reynolds DV. Surgery in the rat during electrical analgesia induced by focal brain stimulation. Science. (1969) 164:4445. doi: $10.1126 /$ science.164.3878.444

4. Eippert F, Bingel U, Schoell ED, Yacubian J, Klinger R, Lorenz $\mathrm{J}$, et al. Activation of the opioidergic descending pain control system underlies placebo analgesia. Neuron. (2009) 63:53343. doi: 10.1016/j.neuron.2009.07.014 though neither sex exhibited neuropathy-induced changes in locomotion, anxiety-, hedonistic-, or depression-like behaviours. Endocannabinoids are widely expressed throughout the DPMS and have an established role in modulating nociceptive transmission. Endocannabinoid levels were measured in key nodes of the DPMS including the prefrontal cortex, periaqueductal grey, rostral ventromedial medulla, and amygdala though no injury dependent changes in endocannabinoid levels were observed at the time-point tested.

In their review article Tavares et al. discuss plasticity of monoaminergic and opioidergic brainstem pathways in preclinical models of pain. Of particular focus is the facilitatory role of the dorsal reticular nucleus, the bi-directional function of the dorsal raphe nuclei, and the inhibitory function of the locus coeruleus. In addition they provide an overview of the opioidergic modulation of these regions. Lastly they discuss the alterations in these signalling systems in models of neuropathic pain and the implications for treatment of patients.

Lastly, in their review article Mills et al. discuss how fMRI studies have provided insight into DPMS function. The main focus is functional connectivity between brainstem nuclei and the spinal cord, and how activity in these networks are disrupted in neuropathy. In addition they discuss the mechanisms that may underlie conditioned pain modulation, and differences in functional connectivity in subjects with and without effective conditioned pain modulation.

\section{AUTHOR CONTRIBUTIONS}

All authors listed have made a substantial, direct and intellectual contribution to the work, and approved it for publication.

\section{ACKNOWLEDGMENTS}

We thank all the authors of the articles collected in this Research Topic. We also thank all the reviewers who have provided the authors with useful suggestions. Finally, we would like to thank all the staff of the Editorial Offices of Frontiers for the great help offered in handling this Research Topic.

5. Willer JC, Le Bars D, De Broucker T. Diffuse noxious inhibitory controls in man: involvement of an opioidergic link. Eur J Pharmacol. (1990) 182:34755. doi: 10.1016/0014-2999(90)90293-F

6. Yarnitsky D, Granot M, Nahman-Averbuch H, Khamaisi M, Granovsky Y. Conditioned pain modulation predicts duloxetine efficacy in painful diabetic neuropathy. Pain. (2012) 153:1193-8. doi: 10.1016/j.pain.2012.02.021

7. Derbyshire SW, Osborn J. Offset analgesia is mediated by activation in the region of the periaqueductal grey and rostral ventromedial medulla. Neuroimage. (2009) 47:1002-6. doi: 10.1016/j.neuroimage.2009.04.032

8. Francois A, Low SA, Sypek EI, Christensen AJ, Sotoudeh C, Beier $\mathrm{KT}$, et al. A brainstem-spinal cord inhibitory circuit for mechanical pain modulation by GABA and Enkephalins. Neuron. (2017) 93:822-39 e6. doi: 10.1016/j.neuron.2017.01.008

9. Oliva V, Hartley-Davies R, Moran R, Pickering AE, Brooks JCW. Simultaneous brain, brainstem and spinal cord pharmacological-fMRI reveals endogenous opioid network interactions mediating attentional analgesia. BioRxiv. (2021) 442823:1-39. doi: 10.1101/2021.05.05.442823 
10. Burgess SE, Gardell LR, Ossipov MH, Malan TP, Jr Vanderah TW, Lai J, et al. Time-dependent descending facilitation from the rostral ventromedial medulla maintains, but does not initiate, neuropathic pain. J Neurosci. (2002) 22:5129-36. doi: 10.1523/JNEUROSCI.22-12-05129.2002

11. De Felice Sanoja R, Wang R, Vera-Portocarrero L, Oyarzo J, King T, Ossipov $\mathrm{MH}$, et al. Engagement of descending inhibition from the rostral ventromedial medulla protects against chronic neuropathic pain. Pain. (2011) 152:27019. doi: 10.1016/j.pain.2011.06.008

12. Hughes SW, Hickey L, Hulse RP, Lumb BM, Pickering AE. Endogenous analgesic action of the pontospinal noradrenergic system spatially restricts and temporally delays the progression of neuropathic pain following tibial nerve injury. Pain. (2013) 154:1680-90. doi: 10.1016/j.pain.2013.05.010

13. Okubo M, Castro A, Guo W, Zou S, Ren K, Wei F, et al. Transition to persistent orofacial pain after nerve injury involves supraspinal serotonin mechanisms. J Neurosci. (2013) 33:5152-61. doi: 10.1523/JNEUROSCI.3390-12.2013

14. Bannister K, Dickenson AH. The plasticity of descending controls in pain: translational probing. J Physiol. (2017) 595:4159-66. doi: 10.1113/JP274165

15. Albusoda A, Ruffle JK, Friis KA, Gysan MR, Drewes AM, Aziz Q, et al. Systematic review with meta-analysis: conditioned pain modulation in patients with the irritable bowel syndrome. Aliment Pharmacol Ther. (2018) 48:797-806. doi: 10.1111/apt.14965

16. Kosek E, Hansson P. Modulatory influence on somatosensory perception from vibration and heterotopic noxious conditioning stimulation (HNCS) in fibromyalgia patients and healthy subjects. Pain. (1997) 70:41-51. doi: 10.1016/S0304-3959(96)03295-2

17. Perrotta A, Serrao M, Ambrosini A, Bolla M, Coppola G, Sandrini $\mathrm{G}$, et al. Facilitated temporal processing of pain and defective supraspinal control of pain in cluster headache. Pain. (2013) 154:1325-32. doi: 10.1016/j.pain.2013.04.012
18. Wilder-Smith OH, Schreyer T, Scheffer GJ, Arendt-Nielsen L. Patients with chronic pain after abdominal surgery show less preoperative endogenous pain inhibition and more postoperative hyperalgesia: a pilot study. J Pain Palliat Care Pharmacother. (2010) 24:119-28. doi: 10.3109/15360281003706069

19. Yarnitsky D, Crispel Y, Eisenberg E, Granovsky Y, Ben-Nun A, Sprecher E, et al. Prediction of chronic post-operative pain: preoperative DNIC testing identifies patients at risk. Pain. (2008) 138:22-8. doi: 10.1016/j.pain.2007.10.033

20. Niesters M, Proto PL, Aarts L, Sarton EY, Drewes AM, Dahan A. Tapentadol potentiates descending pain inhibition in chronic pain patients with diabetic polyneuropathy. Br J Anaesth. (2014) 113:148-56. doi: 10.1093/bja/aeu056

Conflict of Interest: The authors declare that the research was conducted in the absence of any commercial or financial relationships that could be construed as a potential conflict of interest.

Publisher's Note: All claims expressed in this article are solely those of the authors and do not necessarily represent those of their affiliated organizations, or those of the publisher, the editors and the reviewers. Any product that may be evaluated in this article, or claim that may be made by its manufacturer, is not guaranteed or endorsed by the publisher.

Copyright (c) 2021 Patel, Lumb and Bannister. This is an open-access article distributed under the terms of the Creative Commons Attribution License (CC BY). The use, distribution or reproduction in other forums is permitted, provided the original author(s) and the copyright owner(s) are credited and that the original publication in this journal is cited, in accordance with accepted academic practice. No use, distribution or reproduction is permitted which does not comply with these terms. 